\title{
Clinical Observation on the Treatment of Jellyfish Stinging Dermatitis with Qibai Collapse Stains
}

\author{
Huaian $\mathrm{Li}^{*}$, Jin Zhou \\ Qinhuangdao Hospital of Traditional Chinese Medicine, Qinhuangdao 66000, Hebei, China. E-mail: yyx1202yeah.net
}

\begin{abstract}
Objective: To observe the clinical effect on the treatment of jellyfish stinging dermatitis with Qibai collapse stains, and analyze the application value of Qibai collapse stains. Methods: From January 2018 to December 2019, 180 patients with jellyfish stinging dermatitis admitted to our hospital were selected as the research objects. They were randomly divided into experimental group and control group, and the patients in the control group were given routine treatment. Based on the routine treatment, the experimental group was treated combined with external application of Qibai collapse stains ointment. Compared with the therapeutic effect of the two groups, the clinical value in the treatment of jellyfish stinging dermatitis with Qibai collapse stains ointment was analyzed. Results: The total effective rate was $97.8 \%$ in the experimental group and $83.3 \%$ in the control group. The difference between the two groups was statistically significant $(\mathrm{P}<0.05)$. The effect time, swelling time, pain relief time and treatment course in the experimental group were significantly less than those in the control group, with statistically significant differences $(\mathrm{P}<0.05)$. Conclusion: The clinical effect on the treatment of jellyfish stinging dermatitis with Qibai collapse stains is remarkable, which can accelerate the recovery of patients, and is worthy of clinical application.
\end{abstract}

Keywords: Qibai Collapse Stains; Jellyfish Stinging Dermatitis; Clinical Effect

\section{Introduction}

Jellyfish dermatitis is a kind of cnidaria dermatitis. Cnidaria is a unique organelle of cnidaria. When these animals with cnidaria contact the human body, they can prick the cnidaria into the skin and inject the poison into the human body, causing skin damage and inflammation ${ }^{[1]}$. Western medicine treatment of jellyfish stinging dermatitis is mainly anti-allergic, but the effect is not quite ideal. There are studies show that the external effect of traditional Chinese medicine is remarkable ${ }^{[2-3]}$. This work aimed to verify the clinical value on the treatment of jellyfish stinging dermatitis with Qibai collapse stains. Some patients were selected as subjects for a randomized group controlled study. The specific study report is as follows.

\section{Information and methods}

\subsection{General information}

From January 2018 to December 2019, 180 patients with jellyfish stinging dermatitis admitted to our hospital were selected as the research objects. All participants voluntarily participated in the research and signed the informed consent, and the research was approved by the ethics society. The patients were randomly divided into experimental group and control group with 90 patients in each group. In the experimental group, there were 48 males and 42 females, with an average age of $(39.6 \pm 4.5)$

\footnotetext{
Copyright (C) 2020 Huaian Li et al.

doi: $10.18686 /$ aem.v9i1.156

This is an open-access article distributed under the terms of the Creative Commons Attribution Non-Commercial License

(http://creativecommons.org/licenses/by-nc/4.0/), which permits unrestricted non-commercial use, distribution, and reproduction in any medium,

provided the original work is properly cited.
} 
years between $24 \sim 56$ years. In the control group, there were 46 males and 44 females, with an average age of $(39.6 \pm 4.5)$ years between $24 \sim 56$ years. Patients with severe cardiovascular disease, hepatorenal insufficiency and mental disorders were excluded to ensure that the results of the research would not be affected.

\subsection{Methods}

All patients with jellyfish stinging were examined by routine physical examination. Vital signs were measured. Both lungs were normal without dry and wet rales. The patients in the control group were given routine treatment, including thorough removal of residual cnidaria from the skin, and 5\% 10\% sodium bicarbonate solution to wash damaged skin and wet application for $30 \mathrm{~min}$. Also, it includes intramuscular injection of diphenhydramine, promethazine injection, dexamethasone 5-10mg into the pot for once, and vitamin $\mathrm{C}$ calcium gluconate injection for antiallergic treatment. Based on the routine treatment, the patients in the observation group were applied with Qibai collapse stains ointment. The collapse stains ointment was made of Borneol 100g, Borax 100g, raw Phellodendron 100g and raw Astragalus 100g. The ointment was made by mixing powder and vinegar. The ointment was evenly spread on the cotton paper and the thickness was moderate. The spread cotton paper was applied on the affected skin of the patients, and the scope was larger than the damaged skin. It is fixed with breathable adhesive tape once a day for 4-6 hours each time for a course of 5 days.

\subsection{Observation indicators}

The clinical value of Qibai collapse stains ointment was analyzed by comparing the treatment effect, onset time, swelling time, course of treatment and pain relief time between the two groups. Curative effect standard: Cure: the skin swelling pain symptom disappears, and the erythema, blister, papule or itching sensation disappears. Effective: the skin swelling pain symptom alleviates, and the erythema, blister, papule or itching sensation alleviates. Ineffective: the patient's various symptoms slightly or not significantly improved.

\subsection{Observation indicators}

SPSS19.0 software was used for statistical analysis, and $t$ test was used for comparison of measurement data. $\chi 2$ test was used for counting data, and $\mathrm{P}<0.05$ was considered as statistically significant.

\section{Results and discussion}

\subsection{Therapeutic effect of two groups}

The total effective rate was $97.8 \%$ in the experimental group and $83.3 \%$ in the control group. The difference between the two groups was statistically significant $(\mathrm{P}<0.05)$ (See Table 1).

\subsection{Comparison of indicators between the two groups}

The effect time, swelling time, pain relief time and treatment course in the experimental group were significantly less than those in the control group, with statistically significant differences $(\mathrm{P}<0.05)$. (See Table 2)

\begin{tabular}{lllll}
\hline Group & Cure & Effective & Invalid & Total effectiveness \\
\hline Experimental group & $80(88.9)$ & $8(8.9)$ & $2(2.2)$ & $88(97.8)$ \\
Control group & $65(72.2)$ & $10(11.1)$ & $15(16.7)$ & $75(83.3)$ \\
\hline
\end{tabular}

Table 1. Therapeutic effect of two groups $[\mathrm{n}(\%)](\mathrm{n}=90)$

\begin{tabular}{lllll}
\hline Group & Effective time (min) & Swelling time (d) & Course of treatment (d) & Duration of pain relief (d) \\
\hline Experimental group & $5.6 \pm 0.7$ & $3.1 \pm 0.4$ & $5.3 \pm 0.6$ & $2.2 \pm 0.3$ \\
Control group & $20.5 \pm 3.2$ & $6.4 \pm 0.9$ & $8.9 \pm 1.2$ & $4.8 \pm 0.5$ \\
\hline
\end{tabular}

Table 2. Comparative results between the two groups of patients $(n=90)$ 


\section{Discussion}

The skin of jellyfish after stung has electric shock like tingling, numbness, itching and burning sensation. After 4-6 hours, there were linear erythema, papule, wheal, ecchymosis and other dermatitis symptoms. The body has muscle pain, fatigue, chest short, palpitations, shortness of breath, low fever, thirst, cold sweat, etc. A few people are sick and vomiting, abdominal pain, diarrhea, dyspnea, restlessness, blood pressure drop, hemoptysis and phlegm, and they can die of pulmonary edema and allergic rest if rescue is not timely ${ }^{[4-5]}$. Toxic mechanism of jellyfish stinging. There are many tentacles around the mouth and on the shoulder plate of jellyfish, which contain many cnidaria bursa, and the bursa contains the cnidaria toxin. Its main components are protein-like toxin, elastase, carboxypeptidase, strong anesthetics, kinin, vasoactive amines and other vasoactive substances. Once the human skin contacts the tentacles of jellyfish, the cnidaria bursa in it will release the cnidaria toxin, kallikrein and vasoactive amine rapidly. This can make the venules and capillaries expand, the permeability increase, the exudation increase, and the skin hyperemia and edema. Kallikrein and other substances can also produce strong pain, and vasoactive amines can produce itching. Protein-like toxins can cause tissue necrosis, and a large number of cnidaria toxin into the body can cause systemic capillary expansion, sharp increase in permeability, decrease in effective circulation blood volume, and cause hooker.

Due to jellyfish toxin can be neutralized by alkaline solution, traditional western medicine can wash the damaged skin with alkaline solution such as $5 \% \sim 10 \%$ sodium bicarbonate solution or alum water, $1 \%$ ammonia water, and thoroughly remove the prickles in the skin. According to traditional Chinese medicine, jellyfish sting belongs to the acute disease of insect and animal injury poisoning. The poison enters through the skin, restrain pathogenesis, enters the air from the pathogenesis, spreads in the wind and fire, and the heat is full of wind. At present, there is little mention on traditional Chinese medicine in the domestic reports on the treatment of jellyfish sting. Based on the characteristics and pathological basis of jellyfish stinging dermatitis, this hospital made its own Qibai collapse stains ointment. The specific prescription of the drug is Astragalus, Phellodendron, Borneol, Angelica, etc. It is developed into a fine powder. After 120 mesh screening, it is made into an ointment with vinegar or honey, which is ready for use after high-temperature sterilization. According to the theory of internal disease and external treatment in traditional Chinese medicine, the treatment of collapse stains is based on the external treatment method of "bathing by rubbing" proposed in Yellow Emperor's Inner Canon. The traditional Chinese medicine, which can clear away heat, dry dampness, relieve fire, detoxify, diminish inflammation and relieve pain, can be directly absorbed into the skin through the dam stain. This can make the surrounding skin and subcutaneous tissue edema disappear, and relieve pain from the local effect. The Compendium of Materia Medica said that "the main hot wound... insect sores". Modern pharmacological research shows that Phellodendron has antifungal and bacteriostatic effects, and the different berberine contained in the leaves of Phellodendron has anti herpesvirus activity. Astragalus has sweet taste, small temperature, lung and spleen channels, and it has the functions of invigorating Qi and Yang, invigorating Qi and strengthening surface, promoting toxin and muscle, and promoting water and swelling. The Sutra said that "main carbuncle gangrene... discharge pus and relieve pain". The Pearl Sac said that Astragalus have five functions: "... Leaving muscle heat, the fourth, discharge pus and relieve pain, promote blood circulation and generate blood... It is the holy medicine of the sore family ". The modern pharmacological action of Astragalus is antibacterial, antiviral, analgesic and anti-inflammatory, which can increase the collagen synthesis of tissue ${ }^{[6]}$. Borneol has local analgesic and antiseptic inhibitory bacteria effects, and borax has anti-corrosion and protection of skin and mucous membrane effects. Angelica can nourish and activate blood. All drugs are used together to clear away heat and detoxify, promote blood circulation and eliminate swelling, and promote the growth of muscles and relieve pain. In order to verify the effect on the treatment of jellyfish stinging dermatitis with Qibai collapse stains, some patients in the hospital were selected as the image segmentation group contrast study. The data showed that the total effective rate was $97.8 \%$ 
in the experimental group and $83.3 \%$ in the control group. The difference between the two groups was statistically significant $(\mathrm{P}<0.05)$. The effect time, swelling time, pain relief time and treatment course in the experimental group were significantly less than those in the control group, with statistically significant differences $(\mathrm{P}<0.05)$. This indicated that external application of Chinese herbal medicine Qibai collapse stains can significantly improve the treatment effect of jellyfish stinging dermatitis.

In conclusion, Qibai collapse stains ointment has a remarkable effect on the treatment of jellyfish stinging dermatitis, which can obviously shorten the course of treatment and promote the patients to recover more quickly. At the same time, it can alleviate the pain and skin swelling of the patients, which has certain clinical value and is worth popularizing and applying vigorously.

\section{References}

1. Tian C, Zheng X, Wang W. Clinical observation on external application of Qibai collapse stains on pediatric phlebitis (in Chinese). Guangdong Medical Journal 2018; 39(7): 1103-1105. doi: 10.13820/j.cnki.gdyx.2018.07.020.

2. Shang G, Li H, Xiao J. Clinical observation of Qibai collapse stains on treating traumatic dermatitis (in Chinese). Guangdong Medical Journal 2016; 37(15): 2245.

3. Liu W, Su W, Jiang G, et al. Chinese and western medicine treatment of jellyfish dermatitis (in Chinese). Journal of Navy Medicine 2018; 39(6): 584-586. doi: 10.3969/j.issn.1009-0754.2018.06.035.

4. Liu X. Therapeutic effect of cold and wet compress on traditional Chinese medicine for the treatment of jellyfish dermatitis (in Chinese). Guangming Journal of Chinese Medicine 2009; 24(1): 82-83. doi: 10.3969/j.issn.1003-8914.2009.01.058.

5. Hong Y, Zhao L, Wang Y, et al. Analysis on the effect of external application in traditional Chinese medicine Qibai on jellyfish dermatitis stellata (in Chinese). Journal of Hebei TCM and Pharmacology 2018; 33(6): 22- $23+58$.

6. Yang W, Sun Z. Progress in clinical application of traditional Chinese medicine external application (in Chinese). World Medical Information Abstracts 2019; 19(28): 80-81. 